\title{
Topological superfluids and the BEC-BCS crossover in the attractive Haldane-Hubbard model
}

\author{
Yi-Cai Zhang, ${ }^{1}$ Zhihao Xu, ${ }^{2}$ and Shizhong Zhang ${ }^{1, *}$ \\ ${ }^{1}$ Department of Physics, Center of Theoretical and Computational Physics, The University of Hong Kong, Hong Kong, China \\ ${ }^{2}$ Institute of Theoretical Physics, Shanxi University, Taiyuan 030006, China
}

(Received 26 January 2016; published 28 April 2017)

\begin{abstract}
Motivated by the recent realization of the Haldane model in a shaking optical lattice, we investigate the effects of attractive interaction and the BEC-BCS crossover in this model at and away from half-filling. We show that, contrary to the usual $s$-wave BEC-BCS crossover in the lattice, a topological superfluid with Chern number $C=2$ appears in an extended region of the phase space for intermediate strength of the attractive interaction on the interaction-density plane. When inversion symmetry is broken, a gapless weak topological state is realized. We also investigate the fluctuations in these superfluid phases and show that the Anderson-Bogoliubov mode is quadratic due to time-reversal symmetry breaking and the existence of an undamped Leggett mode in the strong-coupling limit. Near the topological phase transition, the damping of the Leggett mode reaches its maximum.
\end{abstract}

DOI: 10.1103/PhysRevA.95.043640

\section{INTRODUCTION}

Cold atoms in optical lattices can be used to simulate important models in condensed-matter physics due to their high controllability and versatility [1,2]. This was demonstrated beautifully by the recent realizations of the Haldane [3-5] and Hofstadter models using a shaking lattice and Raman laser techniques, respectively. In these experiments, the existence of topological bands is verified using Bloch oscillations with either a Bose condensate [6] or free fermions [3]. With tunable interactions in the optical lattices, these advances open new avenues for the controlled study of interaction effects in topological system and pave the way for the possibility of realizing fractional Chern insulators.

Perhaps by far the best-studied interacting topological state is the fractional quantum Hall state in which the strong Coulomb repulsion between electrons generates emergent fractional quasiparticles that obey Abelian or non-Abelian statistics [7-13]. Naturally, with the discovery of topological insulators $[14,15]$, a great deal of effort has been made to investigate its interacting counterparts [16-19]. In the case of the Haldane model, several recent studies have focused on the interplay between magnetic instabilities and possible topological ground states in the case of repulsive interactions [20-24] as well as possible superconducting states with attractive interactions at half-filling [25] (see also Ref. [26]).

In this paper, we consider the analogous nature of the BEC-BCS crossover [27] in the attractive Haldane-Hubbard model. In contrast to the usual BEC-BCS crossover, we found that, away from half-filling, there are extended regions in the parameter space (interaction density) for which a topological superfluid is the ground state. With increasing breaking of inversion symmetry, a gapless weak topological state intervenes before the system enters a trivial superfluid. We also consider the fluctuation effects on the ground states and show that the usual Anderson-Bogoliubov mode becomes quadratic in the absence of time-reversal symmetry and an undamped Leggett mode appears in the strong-coupling limit. Damping of the

\footnotetext{
*shizhong@hku.hk
}

collective modes due to coupling to quasiparticles reaches its maximum as one approaches the phase-transition critical point.

\section{THE HALDANE-HUBBARD MODEL AND ITS SYMMETRY}

Consider a two-component Fermi gas with spin $\sigma=\uparrow, \downarrow$, interacting via an on-site attractive interaction $-U(U>0)$, which can be modeled by the following Haldane-Hubbard model:

$$
\mathcal{H}=\sum_{i j \sigma} t_{i j} c_{i \sigma}^{\dagger} c_{j \sigma}+\sum_{i \sigma}\left(M \epsilon_{i}-\mu\right) n_{i \sigma}-U \sum_{i} n_{i \uparrow} n_{i \downarrow},
$$

where $\mu$ is the chemical potential. $t_{i j}$ is the hopping amplitude between sites $i$ and $j$. For nearest-neighbor hopping, $t_{i j}=t$ and will be set equal to one in the following. For the next-nearest-neighbor hopping $t_{i j}=t^{\prime} \exp (-i \nu \phi)$ with $v=1$ for clockwise hopping and $v=-1$ for anticlockwise hopping [28] (see Fig. 1). Experimentally, $\phi$ is induced by a circular shaking of the optical lattice and, except for $\phi=0, \pi$, will break the time-reversal symmetry. $M$ describes the energy offset of the two sublattices with $\epsilon_{i}=1(-1)$ for the $\mathbf{A}(\mathbf{B})$ sublattices and breaks the inversion symmetry of A-B sublattices. In the following, we use operators $a_{i}^{\dagger}$ and $b_{i}^{\dagger}$ to denote the fermion creation operator at sublattices $\mathbf{A}$ and $\mathbf{B}$, and $i$ now indexes the unit cell, which consists of neighboring $\mathbf{A}$ and $\mathbf{B}$ sites. Now, introducing the three Pauli matrices $\boldsymbol{\tau} \equiv\left(\tau_{x}, \tau_{y}, \tau_{z}\right)$ that describe the sublattice degrees of freedom, the noninteracting Hamiltonian $\mathcal{H}_{0}$ can conveniently be written in momentum space as $\mathcal{H}_{0}(\mathbf{k})=$ $\sum_{\sigma} \psi_{\sigma}^{\dagger}(\mathbf{k}) h(\mathbf{k}) \psi_{\sigma}(\mathbf{k})$, where $\psi_{\sigma}^{\dagger}(\mathbf{k})=\left[a_{\sigma}^{\dagger}(\mathbf{k}), b_{\sigma}^{\dagger}(\mathbf{k})\right]$ and $h(\mathbf{k})=\epsilon(\mathbf{k})+\mathbf{d}(\mathbf{k}) \cdot \boldsymbol{\tau} . \epsilon(\mathbf{k})=2 t^{\prime} \cos \phi \sum_{\delta} \cos \left(\mathbf{k} \cdot \mathbf{b}_{\delta}\right)-\mu$, $d_{x}(\mathbf{k})=t \sum_{\delta} \cos \left(\mathbf{k} \cdot \mathbf{a}_{\delta}\right), d_{y}(\mathbf{k})=-t \sum_{\delta} \sin \left(\mathbf{k} \cdot \mathbf{a}_{\delta}\right)$, and $d_{z}(\mathbf{k})=M-2 t^{\prime} \sin \phi \sum_{\delta} \sin \left(\mathbf{k} \cdot \mathbf{b}_{\delta}\right)$, where $\mathbf{k}=\left(k_{x}, k_{y}\right)$ and the vectors $\mathbf{a}_{\delta}$ and $\mathbf{b}_{\delta}$ are depicted in Fig. 1.

For half-filling and with $U=0$, the system is either a trivial insulator for $|M|>3 \sqrt{3} t^{\prime}|\sin \phi|$ or two copies of the Chern insulator for $|M|<3 \sqrt{3} t^{\prime}|\sin \phi|$ because of spin degeneracy. Away from half-filling, in general, the Fermi surface consists 


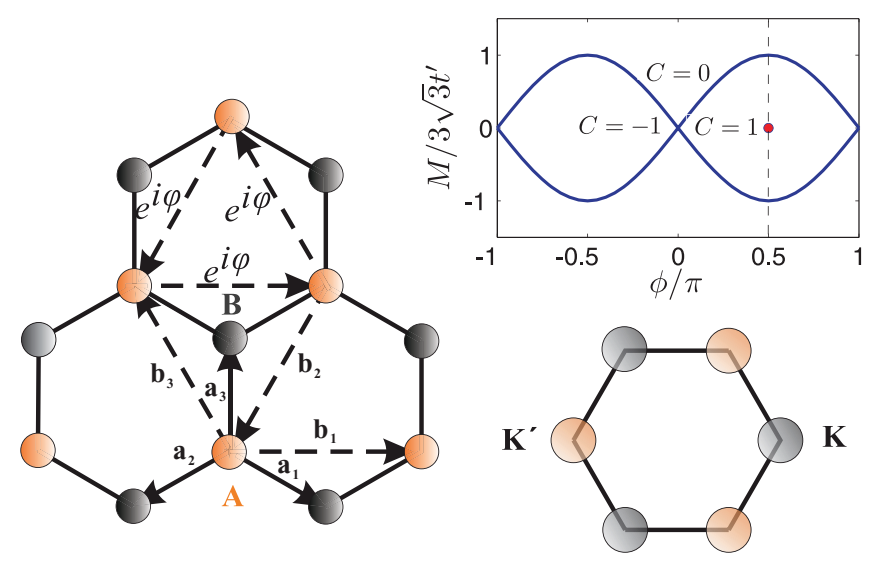

FIG. 1. Panel (a): the honeycomb lattice in the Haldane model. Taking the distance of nearest-neighbor sites as the length unit, here two-dimensional vectors $\mathbf{a}_{1}=[\sqrt{3} / 2,-1 / 2], \mathbf{a}_{2}=[-\sqrt{3} / 2,-1 / 2]$, and $\mathbf{a}_{3}=[0,1] . \mathbf{b}_{1}=[\sqrt{3}, 0], \mathbf{b}_{2}=[-\sqrt{3} / 2,-3 / 2]$, and $\mathbf{b}_{3}=$ $[-\sqrt{3} / 2,3 / 2]$. The red (black) sites denote sublattices A $(\mathbf{B})$. The phase factor $e^{i \phi}$ associated with next-nearest-neighbor anticlock hopping also is labeled. Panel (b) is Haldane's topological phase diagram on the $M-\phi$ plane. Panel (c) shows two Fermi pockets around Dirac points $\mathbf{K}$ and $\mathbf{K}^{\prime}$ in the Brillouin zone (BZ).

of two (particle or hole) pockets located at $\mathbf{K}$ and $\mathbf{K}^{\prime}$, and the system behaves as a metal.

\section{Particle-hole symmetry}

In addition to the $\mathrm{SU}(2)$ spin rotation symmetry of the full Hamiltonian in Eq. (1), for $\phi=\pi / 2$, the Hamiltonian is also invariant under the particle-hole transformation,

$$
c_{i \sigma} \rightarrow \epsilon_{i} c_{i \sigma}^{\dagger}, \quad c_{i \sigma}^{\dagger} \rightarrow \epsilon_{i} c_{i \sigma}
$$

for $M=0$ where we recall $\epsilon_{i}=1(-1)$ for the $\mathbf{A}(\mathbf{B})$ sublattices. Under this transformation, it is easy to see that both the nearest- and the next-nearest-neighbor hopping terms are invariant when $\phi=\pi / 2$. The interaction term remains invariant at half-filling with chemical potential $\mu=0$. On the other hand, when $M \neq 0$, however, one needs to perform an additional inversion transformation $\mathbf{r} \rightarrow-\mathbf{r}$ and an interchange of the $\mathbf{A}$ and $\mathbf{B}$ sublattices in order that the Hamiltonian remains invariant. Thus, for $\phi=\pi / 2$, which will be our focus in the following, it is only necessary to consider the case when $n>2$ (here $n=2$ means two particles per unit cell on average, i.e., half-filling), and the chemical potential satisfies the relation,

$$
\mu(-\Delta n)=-\mu(\Delta n),
$$

where $\Delta n \equiv n-2$ is the deviation from half-filling. As a result, $\mu$ remains zero at half-filling, irrespective of the strength of $U$.

\section{MEAN-FIELD PHASE DIAGRAM AND TOPOLOGICAL SUPERFLUIDS}

To take into account the attractive interactions between opposite spins, we perform the mean-field decoupling in the Cooper channel and introduce $\Delta_{i}=-U\left\langle c_{i \uparrow} c_{i \downarrow}\right\rangle$. In the following, we take the following specific parameters: $t=1$ as the energy unit, $\phi=\pi / 2$, and $t^{\prime}=0.15$. We have checked that, for $M=0, \Delta_{i}$ is uniform and real, in which case, we will denote it as $\Delta$. For $M \neq 0$, however, $\Delta_{i}$ is real but differs for the $\mathbf{A}$ and $\mathbf{B}$ sublattices, and we denote them as $\Delta_{\mathrm{A}}$ and $\Delta_{\mathrm{B}}$, respectively. In the latter case, the Bogoliubov-de Gennes (BdG) equation takes the form

$$
\mathcal{H}_{\mathrm{BdG}}=\sum_{\mathbf{k}} \Psi^{\dagger}(\mathbf{k}) H_{\mathrm{BdG}}(\mathbf{k}) \Psi(\mathbf{k})
$$

with $\Psi^{\dagger}(\mathbf{k})=\left[a_{\uparrow}^{\dagger}(\mathbf{k}), b_{\uparrow}^{\dagger}(\mathbf{k}), a_{\downarrow}(-\mathbf{k}), b_{\downarrow}(-\mathbf{k})\right]$ and

$$
H_{\mathrm{BdG}}=\left[\begin{array}{cccc}
h_{11}(\mathbf{k}) & h_{12}(\mathbf{k}) & \Delta_{\mathrm{A}} & 0 \\
h_{21}(\mathbf{k}) & h_{22}(\mathbf{k}) & 0 & \Delta_{\mathrm{B}} \\
\Delta_{\mathrm{A}}^{*} & 0 & -h_{11}^{*}(-\mathbf{k}) & -h_{12}^{*}(-\mathbf{k}) \\
0 & \Delta_{\mathrm{B}}^{*} & -h_{21}^{*}(-\mathbf{k}) & -h_{22}^{*}(-\mathbf{k})
\end{array}\right] .
$$

Upon diagonalizing $H_{\mathrm{BdG}}$, we obtain the excitation energies given by $E^{p(h) \pm}(\mathbf{k})$, where \pm labels the two branches arising from the underlying A-B sublattices and $p(h)$ denotes the particle (hole) branch due to the particle-hole symmetry of $H_{\mathrm{BdG}}$. The particle-hole symmetry of the BdG Hamiltonian implies $E^{h \pm}(\mathbf{k})=-E^{p \pm}(-\mathbf{k})$. The thermodynamic potential (per unit cell) $\Omega$ at zero temperature is then given by

$$
\Omega=\frac{1}{N} \sum_{\mathbf{k}}\left[2 \epsilon(\mathbf{k})-\left|E^{p+}(\mathbf{k})\right|-\left|E^{p-}(\mathbf{k})\right|\right]+\frac{\left(\Delta_{\mathrm{A}}^{2}+\Delta_{\mathrm{B}}^{2}\right)}{U} .
$$

The mean-field gaps are determined by setting $\partial \Omega / \partial \Delta_{\mathrm{A}, \mathrm{B}}=0$, and the chemical potential $\mu$ is fixed by requiring the average number of particles to be $n=-\partial \Omega / \partial \mu$.

We solve these three equations self-consistently for $\mu$ and $\Delta_{\mathrm{A}, \mathrm{B}}$. To further characterize the mean-field phase diagram, we also compute the following quantities: (1) the Chern number $C$ [29] corresponding to the two quasihole bands with energies $E^{h \pm}(\mathbf{k}),(2)$ the existence or not of the edge states in a finite stripe, and (3) the existence or not of the bulk gap $E_{\text {gap }}$ in the quasiparticle spectrum.

(i) $M=0$. In this case, inversion symmetry is respected, and $\Delta_{i} \equiv \Delta$ is uniform. The phase diagram on the $n-U$ plane is shown in Fig. 2. At half-filling, the system is a Chern insulator with $C=2$ due to spin degeneracy for small attractive interaction (red dashed line in Fig. 2). At a critical value of $U \approx 3.526$, the system enters a topological superfluid state with $C=2$ which, however, occupies only a very small parameter regime before it enters the trivial superfluid with $C=0$ (crossing the phase boundary at $U \approx 3.57$ ). Above half-filling $n>2$, the situation changes dramatically. The noninteracting system is metallic with two Fermi surfaces located around the $\mathbf{K}$ and $\mathbf{K}^{\prime}$ points, which become superfluid due to attractive interactions. When $U$ is either small or large, the superfluid state is trivial and characterized by $C=0$. However, for intermediate attractive interactions, the system enters a topological superfluid state with $C=2$ in a significantly enlarged portion of the phase space as compared to that at half-filling. Inside the topological phase, a bulk gap is always present. In a finite stripe, a pair of edge states with 


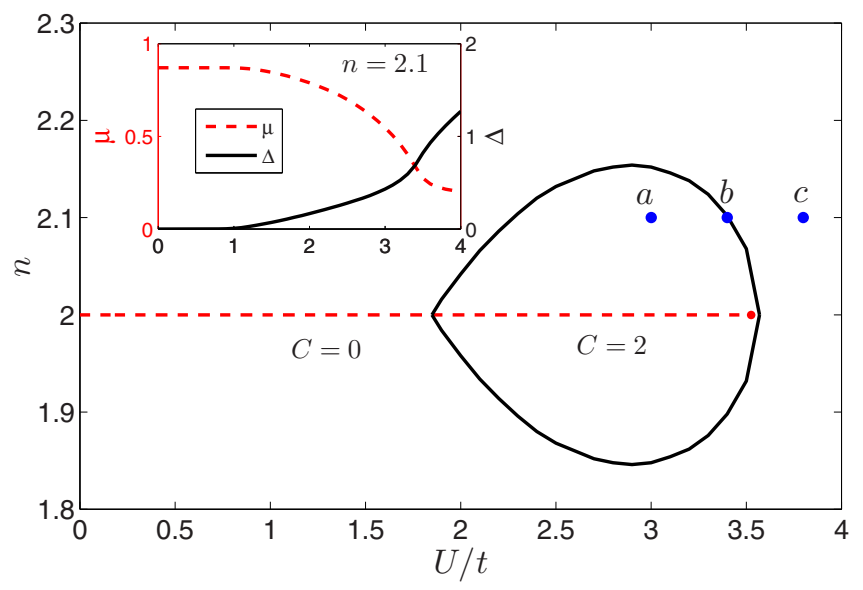

FIG. 2. (a) Phase diagram for $M=0$ on the $n-U$ plane. The region within the black line (except the red dashed line) is in a topological superfluidity phase with Chern number $C=2$. The exterior region of the black line is topologically trivial $(C=0)$. The red dashed line denotes the anomalous quantum Hall insulator $\left(\Delta_{\mathrm{A}(\mathrm{B})} \equiv 0\right)$ at half-filling $(n=2)$. With increasing interaction, e.g., from point $a$ to point $c$, the system crosses the phase boundary. The inset shows chemical $\mu$ potentials and the pairing gaps $\left(\Delta_{\mathrm{A}}=\Delta_{\mathrm{B}}=\right.$ $\Delta$ ) as functions of interaction strength $U$ for filling factor $n=2.1$.

opposite spin exist on both sides of the stripe and carries a net charge current but no spin current as shown in Fig. 3(b).

The main feature of the mean-field phase diagram can be understood from the following observations. It is well known that the topological transition is accompanied by the change in the topology of the energy band and at the transition point the energy gap will close. In the case when $M=0$, the band closing occurs when $3 \sqrt{3} t^{\prime}=\sqrt{\mu^{2}+\Delta^{2}}$ at the $\mathbf{K}$ and $\mathbf{K}^{\prime}$ points. As one increases the interaction $U, \Delta$ increases, but $\mu$ decreases (see the inset of Fig. 2). As a result, in the intermediary value of $U$, the condition $3 \sqrt{3} t^{\prime}>\sqrt{\mu^{2}+\Delta^{2}}$ is satisfied, and a $C=2$ topological state emerges.

(ii) $M \neq 0$. In this case, the inversion symmetry is broken, and $\Delta_{\mathrm{A}} \neq \Delta_{\mathrm{B}}$. In addition to the trivial superfluid state for which $C=0$, there appear two different topological superfluid states. When $|M|$ is small, a fully gapped topological state appears with $C=2$ [Fig. 3(a)], much the same as the case for $M=0$ with a pair of edge states [Fig. 3(c)]. As one increases $M$, a gapless weak topological superfluid state appears when $M=M_{\text {cr1 }}$ for which the bulk gap $E_{\text {gap }}$ becomes zero, whereas the gaps at $\mathbf{K}$ and $\mathbf{K}^{\prime}$ remain finite. This weak topological state is sensitive to disorder which can couple the edge states to the bulk band and thus delocalizes the former. Further increasing $M$, the system enters into a trivial superfluid state when $M=$ $M_{\text {cr2 }}$, the quasiparticle gap $\delta$ at $\mathbf{K}$ or $\mathbf{K}^{\prime}$ closes, and edge states disappear.

Explicitly, for $M>0$, the closing of the bulk gap $E_{\text {gap }}=\min _{k}\left|E^{p-}(k)\right|$ that determines $M_{\text {crl }}$ is given by

$$
3 \sqrt{3} t^{\prime}-\sqrt{\left(\mu+M_{\mathrm{cr} 1}\right)^{2}+\Delta_{\mathrm{B}}^{2}}=0 .
$$

For $M<0$, one only needs to replace $\Delta_{\mathrm{B}}$ with $\Delta_{\mathrm{A}}$. Further increasing $M$, the gaps at $\mathbf{K}$ or $\mathbf{K}^{\prime}$ will close. The critical value (a)
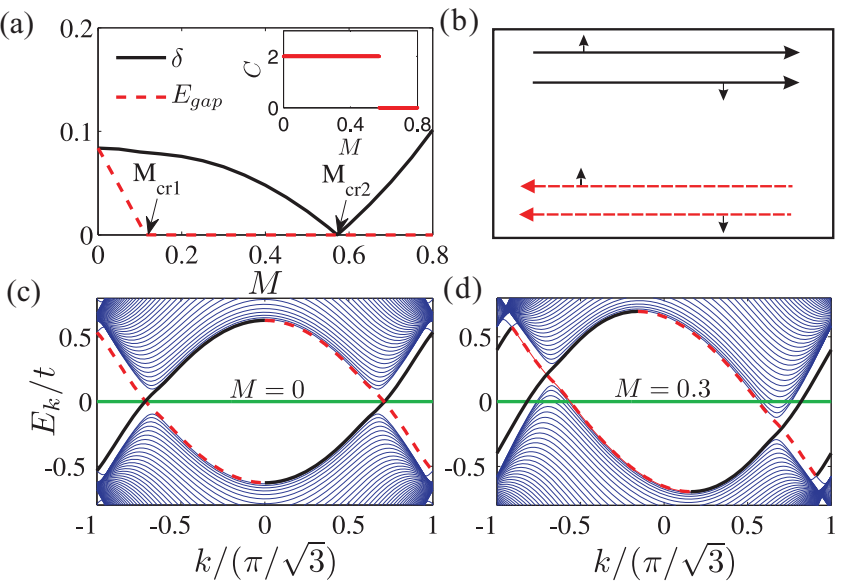

FIG. 3. (a) $E_{\text {gap }}$ and $\delta$ as a function of $M$ for $U=3.0$ (filling $n=2.1$ ). There exist two critical values $M_{\mathrm{cr} 1}=0.1135$ and $M_{\mathrm{cr} 2}=$ 0.5718 . When $M<M_{\text {crl }}$, the bulk gap $E_{\text {gap }}$ remains finite, and the system is in the gapped topological phase. Further increasing $M$ until $M=M_{\text {crl }}, E_{\text {gap }}$ closes, and the system is in the gapless weak topological phase. For $M>M_{\mathrm{cr} 2}$, the system becomes a topologically trivial superfluid. For $M_{\mathrm{cr} 1}<M<M_{\mathrm{cr} 2}$, the system is in the gapless weak topological superfluid state. The Chern number is shown in the inset. (b) Edge states in the boundary of strip geometry. (c) The quasiparticle spectrum (blue) and edge states [red dashed line (black solid line) for left (right) going] in the gapped topological phase for $M=0$. (d) The quasiparticle spectrum (blue) and edge states [red dashed line (black solid line) for left (right) going] in the gapless weak topological phase for $M=0.3$.

of $M_{\mathrm{cr} 2}$ is given by setting $\delta=0$ with

$\delta \equiv 3 \sqrt{3} t^{\prime}-\frac{\sqrt{\left(\mu+M_{\mathrm{cr} 2}\right)^{2}+\Delta_{\mathrm{B}}^{2}}+\sqrt{\left(\mu-M_{\mathrm{cr} 2}\right)^{2}+\Delta_{\mathrm{A}}^{2}}}{2}$.

For $M<0$, we only need to interchange $\Delta_{\mathrm{A}}$ and $\Delta_{\mathrm{B}}$ in Eq. (8). The calculated Chern number $C$ as a function of $M$ is shown in the inset of Fig. 3(a) where it remains at 2 for $M<M_{\mathrm{cr} 2}$.

In the absence of inversion symmetry, it is possible for the system to develop the Fulde-Ferrell-Larkin-Ovchinnikov pairing state $[30,31]$ in which the order parameter $\Delta_{i}$ modulates periodically in real space [32-34]. We have carried out an inhomogeneous Bogoliubov-de Gennes calculation in real space and find no indication of its existence for our chosen parameters. Further inclusion of density wave and stripe orders in our mean-field calculations shows that they are not important in the parameter regime that we have examined (see Appendix A). We note that the $\mathrm{SU}(2)$ spin rotational symmetry is respected, whereas the time-reversal symmetry is broken for our model. Accordingly, the topological superfluidity phases found here belong to class $\mathrm{C}$ in the $\mathrm{BdG}$ class, which are characterized by an even Chern number [35,36]. This is in contrast to the model considered in Ref. [37] where a staggered Zeeman field is present, and thus the spin $\mathrm{SU}(2)$ invariance is broken. 

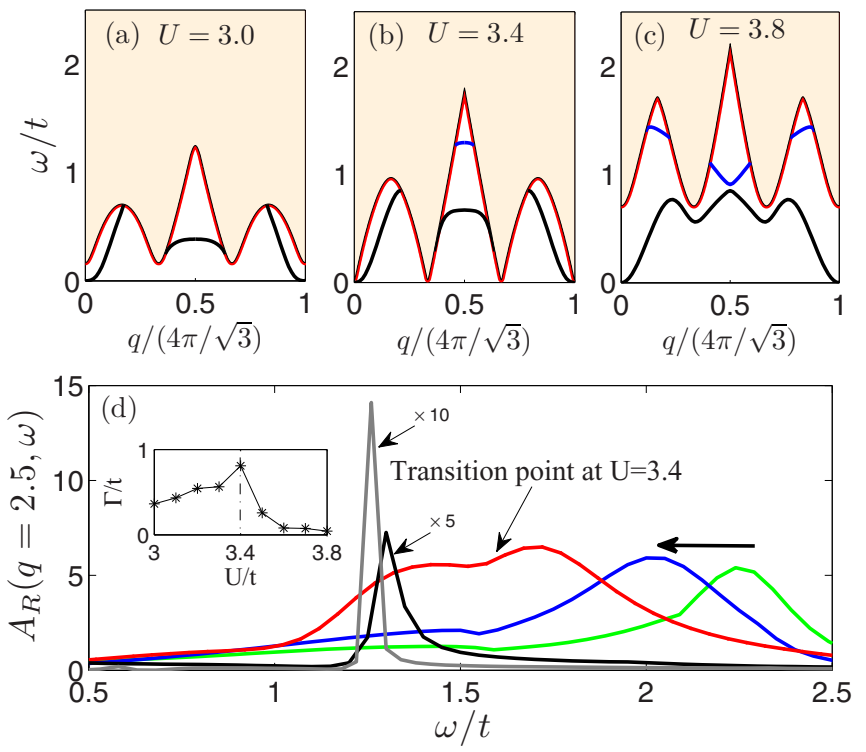

FIG. 4. The collective excitation spectrum for various values of interaction strength $(M=0)$ : (a) $U=3.0$, (b) $U=3.4$, and (c) $U=3.8$, corresponding to the three blue points $a, b$, and $c$, respectively, in Fig. 2. The momentum $\mathbf{q}$ is chosen along the $\hat{x}$ direction $\mathbf{q}=q \hat{x}$. The shaded region denotes the quasiparticle continuum with its lowest boundary (red) given by $\min _{k}\left[E_{k}^{-}+E_{k+q}^{-}\right]$. There are two types of collective excitations. The lower black line corresponds to the gapless Goldstone mode, whereas the upper blue line is the Leggett mode. (d) The spectral function of the Leggett mode for various values of interaction strength $U=3.0,3.2,3.4,3.6,3.8$ (the corresponding peaks are from right to left). Note that the maximal damping occurs at the transition point $(U=3.4)$. The inset shows the variations of the half-width of the spectral function with the interaction strength.

\section{GOLDSTONE MODE, LEGGETT MODE, AND ITS DAMPING}

We now turn to the discussions of collective modes of the attractive Haldane-Hubbard model. For simplicity, we will only consider the situation when $M=0$ and calculate the collective modes by considering Gaussian fluctuations around the mean-field ground state for $n=2.1$ with two Fermi surfaces around $\mathbf{K}$ and $\mathbf{K}^{\prime}$ points (see Fig. 1). To go beyond mean-field theory and calculate the mode frequencies, we expand the order parameters: $\Delta_{\mathrm{A}, \mathrm{B}}(q)=\Delta_{\mathrm{A}, \mathrm{B}}+\eta_{\mathrm{A}, \mathrm{B}}(q)$ and integrate out the fermions to arrive at the effective action for the order parameter fluctuation $S_{\text {eff }}=\frac{1}{2} \sum_{q} \hat{\eta}^{*}(q) M(q) \hat{\eta}(q)$ with $q=\left(\mathbf{q}, i \omega_{n}\right)$ and $\omega_{n}$ as the bosonic Matsubara frequencies. $\hat{\eta}^{*}(q)=\left[\eta_{\mathrm{A}}^{*}(q), \eta_{\mathrm{B}}^{*}(q), \eta_{\mathrm{A}}(-q), \eta_{\mathrm{B}}(-q)\right]$ defines the fluctuation vector in the $\mathbf{A}, \mathbf{B}$ sublattices, and $M(q)$ is the fluctuation matrix whose explicit form is given in Appendix B. The excitation energy is determined by the zeros of its determinant $\operatorname{Det}\left|M\left(\mathbf{q}, i \omega_{n} \rightarrow \omega+i 0^{+}\right)\right|=0$ [38].

Because of the existence of two Fermi surfaces, in addition to the usual Goldstone (or Anderson-Bogoliubov) mode, which corresponds to the oscillation of total density, an additional Leggett mode appears [39], which corresponds to the oscillation of the relative densities of the two Fermi pockets located at the $\mathbf{K}$ and $\mathbf{K}^{\prime}$ points. In Figs. 4(a)-4(c), we show the excitation spectra for three different values of $U$, corresponding to a $C=2$ topological superfluid (point $a$ in Fig. 2), at the phase boundary (point $b$ in Fig. 2) and inside the trivial superfluid (point $c$ in Fig. 2). The shaded region corresponds to the quasiparticle continuum, which touches zero at the transition point, as expected for a quantum phase transition from the topological to the trivial superfluid state. In addition, there are a few further notable features of the collective modes.

First, we note that the long-wavelength Goldstone excitation has a quadratic dispersion $\omega(\mathbf{q})=c|\mathbf{q}|^{2}$, unlike the usual linear dependence, typical of a superfluid [38,40]. This is because, for the Haldane-Hubbard model Eq. (1), time-reversal symmetry is broken, so the low-energy effective theory describing the total phase fluctuation contains the term $\partial_{t} \varphi$ with $\varphi$ as the corresponding fluctuation field. On the other hand, for $M=0$, inversion symmetry is respected, and so the spatial derivative must be of the form $\nabla^{2} \varphi$. Consequently, one finds $\omega(\mathbf{q})=c|\mathbf{q}|^{2}$ at a long wavelength. Furthermore, although the Goldstone mode is always present when $|\mathbf{q}| \rightarrow 0$, it can merge into the quasiparticle continuum and cease to be a well-defined elementary excitation at larger $|\mathbf{q}|$ [see Figs. 4(a) and 4(b)].

Second, we observe that, at strong coupling $(U=3.8)$, a well-defined Leggett mode appears in the first Brillouin zone, whereas at weak coupling, it merges into the continuum and damps away. To investigate the damping of the Leggett mode when it merges into the quasiparticle continuum, we calculate the spectral function of the relative phase fluctuations, $A_{R}(q)=1 / \pi \operatorname{Im}\left\langle\theta^{*}(q) \theta(q)\right\rangle$, where $\theta(q) \equiv \theta_{\mathrm{A}}(q)-$ $\theta_{\mathrm{B}}(q)$ and $\theta_{\mathrm{A}, \mathrm{B}}(q)=\operatorname{Im} \eta_{\mathrm{A}, \mathrm{B}}$. In Fig. 4(d), we show $A_{R}(|\mathbf{q}|=$ $2.5, \omega)$ and found that the damping is maximum at the quantum critical point $U=3.4$. This enhancement is due to the combined effect of the quasiparticle density of states and the enhanced coupling of the Leggett mode to the quasiparticle continuum at the transition.

With the recent realization of the Haldane model in cold atoms, it is possible now to study the topological superfluid states and their quantum phase transitions discussed here. Unlike the noninteracting Haldane model in which the quantum phase transitions can be monitored by using Block oscillation and the band mapping technique, here the phase transition is signaled by the closing of the quasiparticle gap, which will have dramatic effects on the dynamic structure factor that can be measured experimentally with Bragg spectroscopy [41]. The Leggett modes in the multiband superconductor $\mathrm{MgB}_{2}$ have been observed experimentally by tunneling spectroscopy techniques [42], Raman spectroscopy [43], and angle-resolved photoemission spectroscopy [44]. Here we expect that, for neutral atoms, the Leggett modes and their dampings can be measured by the Bragg spectroscopy or modulation spectroscopy.

\section{CONCLUSIONS}

To summarize, away from half-filling for the attractive Haldane-Hubbard model, there exists a gapped nontrivial topological superfluid phase in an extended interaction-density parameter space. When spatial inversion symmetry is broken in the Haldane model, there exists a gapped topological phase to a gapless weak topological phase transition with the increasing in inversion symmetry breaking. In addition, a quadratic dispersion Anderson-Bogliubov mode and a 
gapped Leggett mode appear in such a superfluidic system. Accompanying a phase transition from a topological superfluid to a trivial superfluid, the damping of the Leggett mode reaches its maximum. The damping of the collective mode near the topological phase transition may also be relevant to other topological systems in the condensate matter field, for example, a topological insulator or a superconductor.

\section{ACKNOWLEDGMENTS}

We would like to thank B.-J. Yang for useful discussions. This research was supported by the Hong Kong Research Grants Council (General Research Fund Grant No. HKU 17306414), the Collaborative Research Fund (Grant No. HKUST3/CRF/13G), and the Croucher Foundation under the Croucher Innovation Award.

\section{APPENDIX A: COMPETITION BETWEEN THE DENSITY WAVE AND THE SUPERFLUID PHASE}

In the following, we show that the role of the density wave order is insignificant as compared with superfluid pairing instability in the attractive Haldane-Hubbard model. The density wave order can be written as

$$
n_{i \sigma}-\bar{n}_{i \sigma} \equiv \delta n_{i \sigma}=\sum_{\mathbf{q}} \rho_{\mathbf{q} \sigma} e^{i \mathbf{q} \cdot \mathbf{r}_{i}} .
$$

Here $\sigma=\uparrow / \downarrow$ is the spin index. Furthermore, we assume there is only one component $\chi_{ \pm \mathbf{q} \sigma} \equiv-U\left\langle\rho_{ \pm \mathbf{q} \sigma}\right\rangle \neq 0$, and it satisfies $\chi_{\mathbf{q} \sigma}^{*}=\chi_{-\mathbf{q} \sigma}$. Then the density profile follows $\delta n_{i \sigma} \propto$ $\cos \left(\mathbf{q} \cdot \mathbf{r}_{i}\right)$. The attractive interaction in momentum space is $(U>0)$

$$
V=-U \sum_{\mathbf{q}}\left[\rho_{\mathrm{A}, \mathbf{q} \uparrow}^{\dagger} \rho_{\mathrm{A}, \mathbf{q} \downarrow}+\rho_{\mathrm{B}, \mathbf{q} \uparrow}^{\dagger} \rho_{\mathrm{B}, \mathbf{q} \downarrow}\right],
$$

with $\rho_{\mathrm{Aq} \sigma}=\sum_{\mathbf{k}} a_{\mathbf{k} \sigma}^{\dagger} a_{\mathbf{k}+\mathbf{q} \sigma}, \rho_{\mathrm{Bq} \sigma}=\sum_{\mathbf{k}} b_{\mathbf{k} \sigma}^{\dagger} b_{\mathbf{k}+\mathbf{q} \sigma}$. Due to spin degeneracy, in the following, we assume $\chi_{\mathrm{A}(\mathrm{B}) \mathbf{q}} \equiv$ $\chi_{\mathrm{A}(\mathrm{B}) \mathbf{q} \uparrow}=\chi_{\mathrm{A}(\mathrm{B}) \mathbf{q} \downarrow}$.

In order to perform the calculation in momentum space, we need to assume the momentum $\mathbf{q}$ commensurate with reciprocal vectors $\mathbf{K}$, e.g., $\mathbf{q}=\mathbf{K} / Z$, and here $Z$ is some integer. Due to the density wave order, the order parameter $\chi_{\mathbf{q}}$ couples different unit cells of momentum space. In addition, the superfluid pairing parameters $\Delta_{\mathrm{A}(\mathrm{B})}$ may also be spatially modulated due to density modulation. The pairing amplitude (for sublattice $\mathbf{A}$ ) is

$$
\Delta_{\mathrm{A} i}=-U\left\langle a_{i \downarrow} a_{i \uparrow}\right\rangle=-\frac{U}{N} \sum_{\mathbf{k}, \mathbf{k}^{\prime}}\left\langle a_{\mathbf{k}^{\prime} \downarrow} a_{\mathbf{k} \uparrow}\right\rangle e^{i\left(\mathbf{k}^{\prime}+\mathbf{k}\right) \cdot \mathbf{r}_{i}} .
$$

Furthermore, we assume the pairing amplitude $\Delta_{i}$ shares the same modulation period with density wave $\delta n_{i}$, i.e.,

$$
\begin{aligned}
\Delta_{\mathrm{A}, i+l} & =-\frac{U}{N} \sum_{\mathbf{k}, \mathbf{k}^{\prime}}\left\langle a_{\mathbf{k}^{\prime} \downarrow} a_{\mathbf{k} \uparrow}\right\rangle e^{i\left(\mathbf{k}^{\prime}+\mathbf{k}\right) \cdot \mathbf{r}_{i}+i\left(\mathbf{k}^{\prime}+\mathbf{k}\right) \cdot \mathbf{l}}=\Delta_{\mathrm{A}, i}, \quad \text { (A4) The mean-field equations become }\left(\delta^{\prime}=\delta+6\right) \\
\Delta_{\mathrm{A}} & =\frac{-U}{3 N} \sum_{\mathbf{k} \delta=1,6}\left\{n\left(E_{\delta \mathbf{k}}^{h}\right) u_{1 \delta} u_{3 \delta}^{*}+n\left(E_{\delta \mathbf{k}}^{h}\right) u_{5 \delta} u_{7 \delta}^{*}+n\left(E_{\delta \mathbf{k}}^{h}\right) u_{9 \delta} u_{11 \delta}^{*}+n\left(E_{\delta \mathbf{k}}^{p}\right) u_{1 \delta^{\prime}} u_{3 \delta^{\prime}}^{*}+n\left(E_{\delta \mathbf{k}}^{p}\right) u_{5 \delta^{\prime}} u_{7 \delta^{\prime}}^{*}+n\left(E_{\delta \mathbf{k}}^{p}\right) u_{9 \delta^{\prime}} u_{11 \delta^{\prime}}^{*}\right\}, \\
\Delta_{\mathrm{B}} & =\frac{-U}{3 N} \sum_{\mathbf{k} \delta=1,6}\left\{n\left(E_{\delta \mathbf{k}}^{h}\right) u_{2 \delta} u_{4 \delta}^{*}+n\left(E_{\delta \mathbf{k}}^{h}\right) u_{6 \delta} u_{8 \delta}^{*}+n\left(E_{\delta \mathbf{k}}^{h}\right) u_{10 \delta} u_{12 \delta}^{*}+n\left(E_{\delta \mathbf{k}}^{p}\right) u_{2, \delta^{\prime}} u_{4 \delta^{\prime}}^{*}+n\left(E_{\delta \mathbf{k}}^{p}\right) u_{6 \delta^{\prime}} u_{8 \delta^{\prime}}^{*}+n\left(E_{\delta \mathbf{k}}^{p}\right) u_{10 \delta^{\prime}} u_{12 \delta^{\prime}}^{*}\right\}
\end{aligned}
$$
$e^{i\left(\mathbf{k}^{\prime}+\mathbf{k}\right) \cdot \mathbf{l}}=1$. So we have two possibilities:

(a) $\mathbf{k}^{\prime}+\mathbf{k}=0$ results in the usual gap equation $\Delta_{\mathrm{A}}=$

(b) $\mathbf{k}^{\prime}+\mathbf{k}=\{ \pm 1, \pm 2, \ldots, \pm(Z-1)\} \frac{2 \pi}{l}$ results in the gaps which couple the different sub-bands,

$$
\begin{aligned}
\Delta_{\mathrm{A} 12} & =-\frac{U}{N} \sum_{\mathbf{k}}\left\langle a_{\mathbf{k} \downarrow} a_{-(\mathbf{k}+\mathbf{q}) \uparrow}\right\rangle, \\
\Delta_{\mathrm{A} 13} & =-\frac{U}{N} \sum_{\mathbf{k}}\left\langle a_{\mathbf{k} \downarrow} a_{-(\mathbf{k}+2 \mathbf{q}) \uparrow}\right\rangle, \\
\vdots & \\
\Delta_{\mathrm{A} 1, Z} & =-\frac{U}{N} \sum_{\mathbf{k}}\left\langle a_{\mathbf{k} \downarrow} a_{-(\mathbf{k}+(Z-1) \mathbf{q}) \uparrow}\right\rangle .
\end{aligned}
$$

Because of $\Delta_{1, m}(-\mathbf{q})=\Delta_{1, Z-m+2}(\mathbf{q})$ with $m=2,3, \ldots, Z-1$, $Z$, there are only $Z-1$ extra pairing amplitudes for sublattices A (B). For example, taking $\mathbf{q}=\mathbf{K} / 3$, we need to extend the bases to three unit cells in momentum space with $\psi_{\mathbf{k}}^{\dagger}=\left[a_{\mathbf{k} \uparrow}^{\dagger}, b_{\mathbf{k} \uparrow}^{\dagger}, a_{-\mathbf{k} \downarrow}, b_{-\mathbf{k} \downarrow} ; a_{\mathbf{k}+\mathbf{q} \uparrow}^{\dagger}, b_{\mathbf{k}+\mathbf{q} \uparrow}^{\dagger}, a_{-(\mathbf{k}+\mathbf{q}) \downarrow}, b_{-(\mathbf{k}+\mathbf{q}) \downarrow} ;\right.$ $\left.a_{\mathbf{k}+2 \mathbf{q} \uparrow}^{\dagger}, b_{\mathbf{k}+2 \mathbf{q} \uparrow}^{\dagger}, a_{-(\mathbf{k}+2 \mathbf{q}) \downarrow}, b_{-(\mathbf{k}+2 \mathbf{q}) \downarrow}\right]$. The Hamiltonian then takes following form:

$$
\begin{aligned}
(H)_{12 \times 12} & =\left(\begin{array}{cccc}
H_{\mathrm{BdG}}(\mathbf{k}) & H_{12} & H_{13} \\
H_{12}^{\dagger} & H_{\mathrm{BdG}}(\mathbf{k}+\mathbf{q}) & H_{12} \\
H_{13}^{\dagger} & & H_{12}^{\dagger} & H_{\mathrm{BdG}}(\mathbf{k}+2 \mathbf{q})
\end{array}\right), \\
\left(H_{12}\right)_{4 \times 4} & =\left(\begin{array}{cccc}
\chi_{\mathrm{Aq}}^{*} & 0 & \Delta_{\mathrm{A} 12} & 0 \\
0 & \chi_{\mathrm{Bq}}^{*} & 0 & \Delta_{\mathrm{B} 12} \\
\Delta_{\mathrm{A} 13}^{*} & 0 & -\chi_{\mathrm{Aq}} & 0 \\
0 & \Delta_{\mathrm{B} 13}^{*} & 0 & -\chi_{\mathrm{Bq}}
\end{array}\right), \\
\left(H_{13}\right)_{4 \times 4} & =\left(\begin{array}{cccc}
\chi_{\mathrm{Aq}} & 0 & \Delta_{\mathrm{A} 13} & 0 \\
0 & \chi_{\mathrm{Bq}} & 0 & \Delta_{\mathrm{B} 13} \\
\Delta_{\mathrm{A} 12}^{*} & 0 & -\chi_{\mathrm{A} \mathbf{q}}^{*} & 0 \\
0 & \Delta_{\mathrm{B} 12}^{*} & 0 & -\chi_{\mathrm{Bq}}^{*}
\end{array}\right) .
\end{aligned}
$$

Applying a Bogoliubov transformation $\psi_{\mathbf{k}}^{\dagger}=\tilde{\psi}_{\mathbf{k}}^{\dagger} \hat{O}^{\dagger}$ with a $12 \times 12$ transformation matrix $O_{i j}=u_{i j}$ where $u_{i j}$ are the matrix elements satisfying $\sum_{\delta=1,12}\left|u_{\delta, m}\right|^{2}=1$. The Hamiltonian then is reduced to the diagonal form arranged such that the hole elements appear first, namely,

$$
\begin{aligned}
& \tilde{H}_{i i}=E_{i \mathbf{k}}^{h} \quad(1 \leqslant i \leqslant 6), \\
& \tilde{H}_{i i}=E_{(i-6) \mathbf{k}}^{p} \quad(7 \leqslant i \leqslant 12) .
\end{aligned}
$$

with $l=2 \pi / q$. Comparing the above two equations, we get $-\frac{U}{N} \sum_{\mathbf{k}}\left\langle a_{\mathbf{k} \downarrow} a_{-\mathbf{k} \uparrow}\right\rangle$. 


$$
\begin{aligned}
& \Delta_{\mathrm{A} 12}=\frac{-U}{3 N} \sum_{\mathbf{k} \delta=1,6}\left\{n\left(E_{\delta \mathbf{k}}^{h}\right) u_{1 \delta} u_{7 \delta}^{*}+n\left(E_{\delta \mathbf{k}}^{h}\right) u_{5 \delta} u_{11 \delta}^{*}+n\left(E_{\delta \mathbf{k}}^{h}\right) u_{9 \delta} u_{3 \delta}^{*}+n\left(E_{\delta \mathbf{k}}^{p}\right) u_{1 \delta^{\prime}} u_{7 \delta^{\prime}}^{*}+n\left(E_{\delta \mathbf{k}}^{p}\right) u_{5 \delta} u_{11 \delta^{\prime}}^{*}+n\left(E_{\delta k}^{p}\right) u_{9 \delta^{\prime}} u_{3 \delta^{\prime}}^{*}\right\}, \\
& \Delta_{\mathrm{A} 13}=\frac{-U}{3 N} \sum_{\mathbf{k} \delta=1,6}\left\{n\left(E_{\delta \mathbf{k}}^{h}\right) u_{1 \delta} u_{11 \delta}^{*}+n\left(E_{\delta \mathbf{k}}^{h}\right) u_{5 \delta} u_{3 \delta}^{*}+n\left(E_{\delta \mathbf{k}}^{h}\right) u_{9 \delta} u_{7 \delta}^{*}+n\left(E_{\delta \mathbf{k}}^{p}\right) u_{1 \delta^{\prime}} u_{11 \delta^{\prime}}^{*}+n\left(E_{\delta \mathbf{k}}^{p}\right) u_{5 \delta^{\prime}} u_{3 \delta^{\prime}}^{*}+n\left(E_{\delta \mathbf{k}}^{p}\right) u_{9 \delta^{\prime}} u_{7 \delta^{\prime}}^{*}\right\}, \\
& \Delta_{\mathrm{B} 12}=\frac{-U}{3 N} \sum_{\mathbf{k} \delta=1,6}\left\{n\left(E_{\delta \mathbf{k}}^{h}\right) u_{2 \delta} u_{8 \delta}^{*}+n\left(E_{\delta \mathbf{k}}^{h}\right) u_{6 \delta} u_{12 \delta}^{*}+n\left(E_{\delta \mathbf{k}}^{h}\right) u_{10 \delta} u_{4 \delta}^{*}+n\left(E_{\delta \mathbf{k}}^{p}\right) u_{2 \delta^{\prime}} u_{8 \delta^{\prime}}^{*}+n\left(E_{\delta \mathbf{k}}^{p}\right) u_{6 \delta^{\prime}} u_{12 \delta^{\prime}}^{*}+n\left(E_{\delta \mathbf{k}}^{p}\right) u_{10 \delta^{\prime}} u_{4 \delta^{\prime}}^{*}\right\}, \\
& \Delta_{\mathrm{B} 13}= \frac{-U}{3 N} \sum_{\mathbf{k} \delta=1,6}\left\{n\left(E_{\delta \mathbf{k}}^{h}\right) u_{2 \delta} u_{12 \delta}^{*}+n\left(E_{\delta \mathbf{k}}^{h}\right) u_{6 \delta} u_{4 \delta}^{*}+n\left(E_{\delta \mathbf{k}}^{h}\right) u_{10 \delta} u_{8 \delta}^{*}+n\left(E_{\delta \mathbf{k}}^{p}\right) u_{2 \delta^{\prime}} u_{12 \delta^{\prime}}^{*}+n\left(E_{\delta \mathbf{k}}^{p}\right) u_{6 \delta} u_{4 \delta^{\prime}}^{*}+n\left(E_{\delta \mathbf{k}}^{p}\right) u_{10 \delta^{\prime}} u_{\delta \delta^{\prime}}^{*}\right\}, \\
& \chi_{\mathrm{Aq}}=\frac{-U}{3 N} \sum_{\mathbf{k} \delta=1,6}\left\{n\left(E_{\delta \mathbf{k}}^{h}\right) u_{1 \delta}^{*} u_{5 \delta}+n\left(E_{\delta \mathbf{k}}^{h}\right) u_{5 \delta}^{*} u_{9 \delta}+n\left(E_{\delta \mathbf{k}}^{h}\right) u_{9 \delta}^{*} u_{1 \delta}+n\left(E_{\delta \mathbf{k}}^{p}\right) u_{1 \delta^{\prime}}^{*} u_{5 \delta^{\prime}}+n\left(E_{\delta \mathbf{k}}^{p}\right) u_{5 \delta^{\prime}}^{*} u_{9 \delta^{\prime}}+n\left(E_{\delta \mathbf{k}}^{p}\right) u_{9 \delta^{\prime}}^{*} u_{1 \delta^{\prime}}\right\}, \\
& \chi_{\mathrm{Bq}}=\frac{-U}{3 N} \sum_{\mathbf{k} \delta=1,6}\left\{n\left(E_{\delta \mathbf{k}}^{h}\right) u_{2 \delta}^{*} u_{6 \delta}+n\left(E_{\delta \mathbf{k}}^{h}\right) u_{6 \delta}^{*} u_{10 \delta}+n\left(E_{\delta \mathbf{k}}^{h}\right) u_{10 \delta}^{*} u_{2 \delta}+n\left(E_{\delta \mathbf{k}}^{p}\right) u_{2 \delta^{\prime}}^{*} u_{6 \delta^{\prime}}+n\left(E_{\delta \mathbf{k}}^{p}\right) u_{6 \delta^{\prime}}^{*} u_{10 \delta^{\prime}}+n\left(E_{\delta \mathbf{k}}^{p}\right) u_{10 \delta^{\prime}}^{*} u_{2 \delta^{\prime}}\right\},
\end{aligned}
$$

where $n(E)=\frac{1}{e^{\beta E}+1}$ is the Fermi distribution function with $\beta=1 / T$ and if $E^{h}<0, n\left(E^{h}\right)=1-n\left(\left|E^{h}\right|\right)$. The appearance of factor $1 / 3$ is because the summations over $k$ repeat three times in momentum space (we sum over $k$ in the original Brillouin zone, whereas the Brillouin zone with density modulation is $1 / 3$ of the original one). At $T=0$, we take $\beta \rightarrow \infty$. The number equation is

$$
\begin{aligned}
n= & \frac{2}{3 N} \sum_{\mathbf{k} \delta=1,2, \ldots, 6 ; m=0,4,8}\left\{n\left(E_{\delta k}^{h}\right)\left[\left|u_{1+m \delta}\right|^{2}+\left|u_{2+m \delta}\right|^{2}\right]\right. \\
& \left.+n\left(E_{\delta k}^{p}\right)\left[\left|u_{1+m \delta^{\prime}}\right|^{2}+\left|u_{2+m \delta^{\prime}}\right|^{2}\right]\right\},
\end{aligned}
$$

where the factor 2 arises from the spin degeneracy and $\delta^{\prime}=\delta+6$.

The results of our calculation are shown in Fig. 5 where we take $t=1, t^{\prime}=0.15, U=3, n=2.1$ as in the paper. Figure 5 shows two typical mean-field parameters $\Delta$ 's and $\chi$ 's
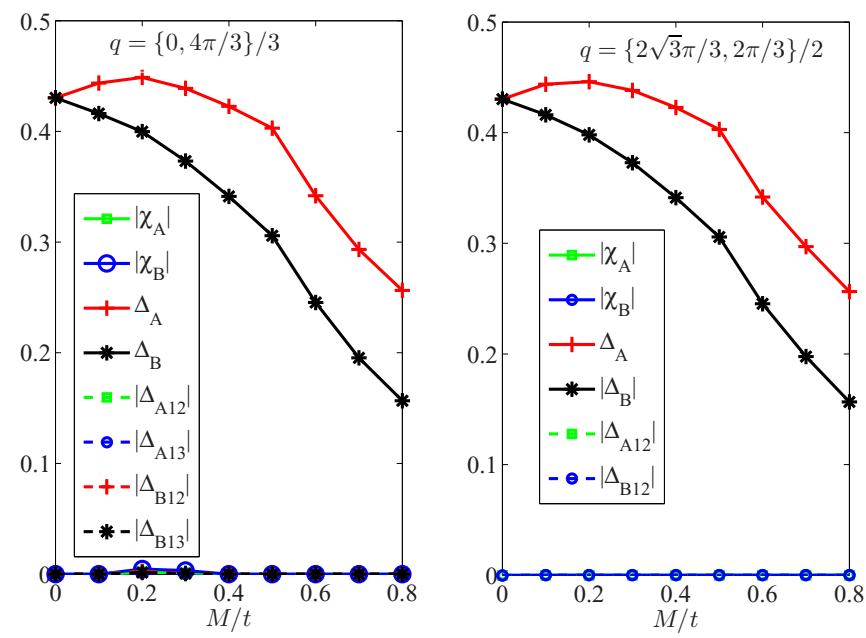

FIG. 5. The variations of order parameters with $M$ at $U=3$. For our chosen parameters, the order parameters for the density wave are always very small. as a function of $M$ for $\mathbf{q}=\{0,4 \pi / 3\} / 3$ (left panel) and $\mathbf{q}=$ $\{2 \sqrt{2} \pi / 3,2 \pi / 3\} / 2$ (right panel). We can see the parameters of density wave order $\chi$ and other pairing parameters are very small for most of the cases, e.g., $\left|\chi / \Delta_{\mathrm{A} / \mathrm{B}}\right|<10^{-4}$. Only when $M=0.2,0.3$, are the parameters a bit enhanced $\left|\chi / \Delta_{\mathrm{A} / \mathrm{B}}\right| \sim 10^{-2}$ (the left panel). So the effects of the density wave orders in the Haldane model are not evident. The pairing superfluid orders are still stable against density wave orders as a whole. One way to understand this result is that, in our model, there is no next-nearest-neighbor interaction, which in the case of the standard Hubbard model would have favored density orders. Furthermore, there is no nesting in our model, so charge-density wave instability is not particularly enhanced.

\section{APPENDIX B: FLUCTUATIONS AND THE COLLECTIVE MODE}

To investigate the collective mode and its damping, it is most straightforward to formulate the theory in functional integration. The partition function in the grand canonical ensemble is written as a functional integral of Grassman fields $\bar{\psi}$ and $\psi[40]$,

$$
Z \equiv \int D \bar{\psi}_{\mathrm{A} i \sigma}(\tau) D \psi_{\mathrm{A} i \sigma}(\tau) D \bar{\psi}_{\mathrm{B} j \sigma}(\tau) D \psi_{\mathrm{B} j \sigma}(\tau) e^{-S},
$$

where we have the action $S$ given by

$$
\begin{aligned}
S= & \sum_{\mathrm{A} i, \sigma ; \mathrm{B} j, \sigma} \int_{0}^{\beta} d \tau\left[\bar{\psi}_{\mathrm{A} i \sigma}(\tau) \frac{\partial \psi_{\mathrm{A} i \sigma}(\tau)}{\partial \tau}\right. \\
& \left.+\bar{\psi}_{\mathrm{B} j \sigma}(\tau) \frac{\partial \psi_{\mathrm{B} j \sigma}(\tau)}{\partial \tau}+H\left(\psi_{\mathrm{A}}, \psi_{\mathrm{B}}\right)\right],
\end{aligned}
$$

where $\beta=1 / T$. In this paper, we will only focus on the properties at zero temperature and will take $\beta \rightarrow \infty$ at the end. In the following, it is assumed that the subscript $i$ or $i^{\prime}(j$ or $\left.j^{\prime}\right)$ always denotes the position of sublattices $\mathbf{A}(\mathbf{B})$ unless stated otherwise. 
By introducing the Hubbard-Stratanovich fields $\Delta_{\mathrm{A}(\mathrm{B}) i}$ for sublattices $\mathbf{A}(\mathbf{B})$ as usual, we have

$$
Z=\int D \Delta^{*} D \Delta D \bar{\psi} D \psi e^{-S_{\Delta}}
$$

and the effective action is given by

$$
S_{\Delta}=\int_{0}^{\beta} d \tau \sum_{\mathrm{A} i, \sigma ; \mathrm{B} j, \sigma}\left[\frac{\left|\Delta_{\mathrm{A} i \sigma}(\tau)\right|^{2}}{U}+\frac{\left|\Delta_{\mathrm{B} j \sigma}(\tau)\right|^{2}}{U}-\bar{\psi} G^{-1}\left(\Delta_{\mathrm{A} i}(\tau), \Delta_{\mathrm{B} j}(\tau)\right) \psi\right] .
$$

In the basis $\bar{\psi}=\left[\cdots \bar{\psi}_{\mathrm{A} i \uparrow} \cdots \bar{\psi}_{\mathrm{B} j \uparrow}, \ldots ; \ldots \psi_{\mathrm{A} i \downarrow} \cdots \psi_{\mathrm{B} j \downarrow}, \ldots\right]$, the inverse Nambu-Gorkov Green's function $G^{-1}$ takes following form:

$$
G^{-1}=\left(\begin{array}{cccc}
(A)_{i, i^{\prime}} & (A)_{i, j} & (B)_{i, i^{\prime}} & 0 \\
(A)_{j, i} & (A)_{j, j^{\prime}} & 0 & (B)_{j, j^{\prime}} \\
(B)_{i, i^{\prime}}^{*} & 0 & (D)_{i, i^{\prime}} & (D)_{i, j} \\
0 & (B)_{j, j^{\prime}}^{*} & (D)_{j, i} & (D)_{j, j^{\prime}}
\end{array}\right)
$$

where

$$
\begin{aligned}
A_{i, i^{\prime}} & =\left(-\partial_{\tau}-M+\mu\right) \delta_{i, i^{\prime}} \delta\left(\tau-\tau^{\prime}\right)-t^{\prime} \delta\left(\tau-\tau^{\prime}\right) \sum_{\delta}\left[\delta_{i, i^{\prime}-\mathbf{b}_{\delta}} e^{i \phi}+\delta_{i, i^{\prime}+\mathbf{b}_{\delta}} e^{-i \phi}\right], \\
A_{i, j} & =-t \sum_{\delta} \delta_{i, j-\mathbf{a}_{\delta}} \delta\left(\tau-\tau^{\prime}\right), \quad A_{j, i}=-t \sum_{\delta} \delta_{j, i+\mathbf{a}_{\delta}} \delta\left(\tau-\tau^{\prime}\right), \\
A_{j, j^{\prime}} & =\left(-\partial_{\tau}+M+\mu\right) \delta_{j, j^{\prime}} \delta\left(\tau-\tau^{\prime}\right)-t^{\prime} \delta\left(\tau-\tau^{\prime}\right) \sum_{\delta}\left[\delta_{j, j^{\prime}-\mathbf{b}_{\delta}} e^{-i \phi}+\delta_{j, j^{\prime}+\mathbf{b}_{\delta}} e^{i \phi}\right], \\
B_{i, i^{\prime}} & =\Delta_{\mathrm{A} i} \delta_{i, i^{\prime}} \delta\left(\tau-\tau^{\prime}\right), \quad B_{j, j^{\prime}}=\Delta_{\mathrm{B} j} \delta_{j, j^{\prime}} \delta\left(\tau-\tau^{\prime}\right), \\
D_{i, i^{\prime}} & =\left(-\partial_{\tau}+M-\mu\right) \delta_{i, i^{\prime}} \delta\left(\tau-\tau^{\prime}\right)+t^{\prime} \delta\left(\tau-\tau^{\prime}\right) \sum_{\delta}\left[\delta_{i, i^{\prime}-\mathbf{b}_{\delta}} e^{-i \phi}+\delta_{i, i^{\prime}+\mathbf{b}_{\delta}} e^{i \phi}\right], \\
D_{i, j} & =t \sum_{\delta} \delta_{i, j-\mathbf{a}_{\delta}} \delta\left(\tau-\tau^{\prime}\right), \quad D_{j, i}=t \sum_{\delta} \delta_{i, j+\mathbf{a}_{\delta}} \delta\left(\tau-\tau^{\prime}\right), \\
D_{j, j^{\prime}} & =\left(-\partial_{\tau}-M-\mu\right) \delta_{j, j^{\prime}} \delta\left(\tau-\tau^{\prime}\right)+t^{\prime} \delta\left(\tau-\tau^{\prime}\right) \sum_{\delta}\left[\delta_{j, j^{\prime}-\mathbf{b}_{\delta}} e^{i \phi}+\delta_{j, j^{\prime}+\mathbf{b}_{\delta}} e^{-i \phi}\right] .
\end{aligned}
$$

The functional integral is quadratic with respect to the $\psi$ fields, and we can integrate them out,

$$
Z=\int D \Delta^{*} D \Delta \exp \left\{-\int_{0}^{\beta} d \tau \sum_{i, j}\left[\frac{\left|\Delta_{\mathrm{A} i}(\tau)\right|^{2}}{U}+\frac{\left|\Delta_{\mathrm{B} j}(\tau)\right|^{2}}{U}-\operatorname{Tr} \ln G^{-1}\left(\Delta_{\mathrm{A} i}(\tau), \Delta_{\mathrm{B} j}(\tau)\right)\right]\right\} .
$$

Assuming $\Delta_{\mathrm{A} i}$ and $\Delta_{\mathrm{B} j}$ can be written as $\Delta_{\mathrm{A} i}(\tau)=\Delta_{\mathrm{A}}+\eta_{\mathrm{A} i}(\tau)$ and $\Delta_{\mathrm{B} j}(\tau)=\Delta_{\mathrm{B}}+\eta_{\mathrm{B} j}(\tau)$, here $\Delta_{\mathrm{A}(\mathrm{B})}$ 's are not dependent on spatial and time variables. It is convenient to write $G^{-1}$ in momentum and Matsubara frequency spaces applying a Fourier transformation $\psi(i, \tau)=\frac{1}{(2 \pi)^{2} \beta} \sum_{n} \int_{\mathrm{BZ}} d^{2} \vec{k} e^{i \mathbf{k} \cdot \mathbf{r}_{i}-i \omega_{n} \tau} \psi\left(\mathbf{k}, i \omega_{n}\right)$ with $\omega_{n}=(2 n+1) \pi / \beta$ for the Grassman variable, one can get

$$
G^{-1}\left(\mathbf{k}, \mathbf{k}^{\prime}\right)=G_{0}^{-1}\left(\mathbf{k}, \mathbf{k}^{\prime}\right)+K\left(\mathbf{k}, \mathbf{k}^{\prime}\right)
$$

where

$$
G_{0}^{-1}\left(\mathbf{k}, \mathbf{k}^{\prime}\right)=\left(\begin{array}{cccc}
A_{1,1} & A_{1,2} & \Delta_{\mathrm{A}} & 0 \\
A_{2,1} & A_{2,2} & 0 & \Delta_{\mathrm{B}} \\
\Delta_{\mathrm{A}}^{*} & 0 & D_{1,1} & D_{1,1} \\
0 & \Delta_{\mathrm{B}}^{*} & D_{2,1} & D_{2,2}
\end{array}\right) \delta\left(\mathbf{k}-\mathbf{k}^{\prime}\right)
$$

and

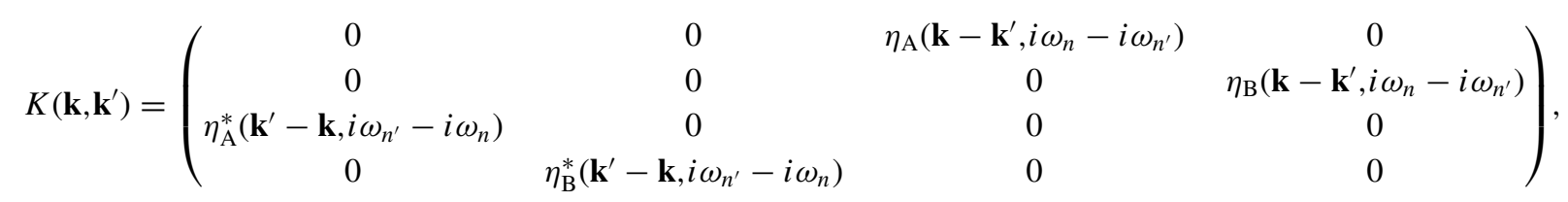


where we introduce $\delta\left(\mathbf{k}-\mathbf{k}^{\prime}\right) \equiv \delta^{2}\left(\mathbf{k}-\mathbf{k}^{\prime}\right) \delta_{n, n^{\prime}}$ and

$$
\begin{aligned}
& A_{1,1}=i \omega_{n}-M+\mu-2 t_{2} \sum_{\delta} \cos \left(\mathbf{k} \cdot \mathbf{b}_{\delta}+\phi\right), \quad A_{1,2}=-t_{1} \sum_{\delta} e^{i \mathbf{k} \cdot \mathbf{a}_{\delta}}, \quad A_{2,1}=\left(A_{1,2}\right)^{*}, \\
& A_{2,2}=i \omega_{n}+M+\mu-2 t_{2} \sum_{\delta} \cos \left(\mathbf{k} \cdot \mathbf{b}_{\delta}-\phi\right), \quad D_{1,1}=i \omega_{n}+M-\mu+2 t_{2} \sum_{\delta} \cos \left(\mathbf{k} \cdot \mathbf{b}_{\delta}-\phi\right), \\
& D_{1,2}=t_{1} \sum_{\delta} e^{i \mathbf{k} \cdot \mathbf{a}_{\delta}}, \quad D_{2,1}=\left(D_{1,2}\right)^{*}, \quad D_{2,2}=i \omega_{n}-M-\mu+2 t_{2} \sum_{\delta} \cos \left(\mathbf{k} \cdot \mathbf{b}_{\delta}+\phi\right) .
\end{aligned}
$$

If we neglect fluctuation terms $\eta_{\mathrm{A} i(\mathrm{~B} j)}(\tau)$, then we obtain the mean-field equations given in the main text. Expanding action $S_{\Delta}$ to the second order of $\eta$, one gets the thermodynamic potential,

$$
Z \approx e^{-S_{0}} \int D \bar{\eta}_{q} D \eta_{q} e^{-S_{\eta}}
$$

where $S_{0}$ is the action from the mean-field contribution. The fluctuation's contribution is

$$
S_{\eta}=\frac{1}{2} \sum_{\mathbf{q}, n} \overline{\eta_{q}} M \eta_{q}
$$

where

$$
\bar{\eta}_{q}=\left[\eta_{\mathrm{A}}^{*}\left(\mathbf{q}, i \omega_{n}\right), \eta_{\mathrm{B}}^{*}\left(\mathbf{q}, i \omega_{n}\right), \eta_{\mathrm{A}}\left(-\mathbf{q},-i \omega_{n}\right), \eta_{\mathrm{B}}\left(-\mathbf{q},-i \omega_{n}\right)\right],
$$

and the fluctuation matrix $M$ is given by

$$
\begin{aligned}
& M_{1 m}\left(\mathbf{q}, i \omega_{n}\right)=\frac{1}{\beta} \sum_{\mathbf{k}, n^{\prime}}\left(G_{13}^{0}+G_{23}^{0}+G_{33}^{0}+G_{43}^{0}\right) G_{1 m}^{0^{\prime}}+\frac{1}{U} \delta_{1, m}, \\
& M_{2 m}\left(\mathbf{q}, i \omega_{n}\right)=\frac{1}{\beta} \sum_{\mathbf{k}, n^{\prime}}\left(G_{14}^{0}+G_{24}^{0}+G_{34}^{0}+G_{44}^{0}\right) G_{2 m}^{0^{\prime}}+\frac{1}{U} \delta_{2, m}, \\
& M_{3 m}\left(\mathbf{q}, i \omega_{n}\right)=\frac{1}{\beta} \sum_{\mathbf{k}, n^{\prime}}\left(G_{11}^{0}+G_{21}^{0}+G_{31}^{0}+G_{41}^{0}\right) G_{3 m}^{0^{\prime}}+\frac{1}{U} \delta_{3, m}, \\
& M_{4 m}\left(\mathbf{q}, i \omega_{n}\right)=\frac{1}{\beta} \sum_{\mathbf{k}, n^{\prime}}\left(G_{12}^{0}+G_{22}^{0}+G_{32}^{0}+G_{42}^{0}\right) G_{4 m}^{0^{\prime}}+\frac{1}{U} \delta_{4, m},
\end{aligned}
$$

where the Green's functions are $G^{0} \equiv\left(G^{0}\left(\mathbf{k}, i \omega_{n^{\prime}}\right)\right)_{4 \times 4}$ and $G^{0^{\prime}} \equiv G^{0}\left(\mathbf{k}+\mathbf{q}, i \omega_{n^{\prime}}+i \omega_{n}\right), m=1-4$. The collective modes are given by zeros of determinant $\operatorname{Det}\left|M\left(\mathbf{q}, i \omega_{n} \rightarrow \omega+i 0^{+}\right)\right|=0$. Introducing amplitudes and phases fields $\eta_{\mathrm{A}}\left(\mathbf{q}, i \omega_{n}\right)=$ $\left[\lambda_{\mathrm{A}}\left(\mathbf{q}, i \omega_{n}\right)+i \theta_{\mathrm{A}}\left(\mathbf{q}, i \omega_{n}\right)\right] / \sqrt{2}$ and $\eta_{\mathrm{B}}\left(\mathbf{q}, i \omega_{n}\right)=\left[\lambda_{\mathrm{B}}\left(\mathbf{q}, i \omega_{n}\right)+i \theta_{\mathrm{B}}\left(\mathbf{q}, i \omega_{n}\right)\right] / \sqrt{2}$, total (relative) amplitudes $\lambda_{1}=\left[\lambda_{\mathrm{A}}\left(\mathbf{q}, i \omega_{n}\right)+\right.$ $\left.\lambda_{\mathrm{B}}\left(\mathbf{q}, i \omega_{n}\right)\right] / \sqrt{2}\left(\lambda_{2}=\left[\lambda_{\mathrm{A}}\left(\mathbf{q}, i \omega_{n}\right)-\lambda_{\mathrm{B}}\left(\mathbf{q}, i \omega_{n}\right)\right] / \sqrt{2}\right)$, and total (relative) phases $\theta_{1}=\left[\theta_{\mathrm{A}}\left(\mathbf{q}, i \omega_{n}\right)+\theta_{\mathrm{B}}\left(\mathbf{q}, i \omega_{n}\right)\right] / \sqrt{2}\left(\theta_{2}=\right.$ $\left.\left[\theta_{\mathrm{A}}\left(\mathbf{q}, i \omega_{n}\right)-\theta_{\mathrm{B}}\left(\mathbf{q}, i \omega_{n}\right)\right] / \sqrt{2}\right)$, in the basis $\left[\lambda_{1}, \lambda_{2}, \theta_{1}, \theta_{2}\right]$, the matrix $(M)_{4 \times 4}$ becomes $M^{\prime}=U^{+} M U$, where

$$
U=\frac{1}{2}\left(\begin{array}{rrrr}
1 & 1 & i & i \\
1 & -1 & i & -i \\
1 & 1 & -i & -i \\
1 & -1 & -i & i
\end{array}\right)
$$

After integrating out amplitudes $\lambda_{1}(\mathbf{q})$ and $\lambda_{2}(\mathbf{q})$, one can get effective $2 \times 2$ fluctuation matrices for phases $\theta_{1(2)}$, i.e., $M^{\prime \prime}=$ $D-C A^{-1} B$, where the $2 \times 2$ submatrices are as follows:

$$
\begin{array}{ll}
A=\left(\begin{array}{ll}
M_{11}^{\prime} & M_{12}^{\prime} \\
M_{21}^{\prime} & M_{22}^{\prime}
\end{array}\right), & B=\left(\begin{array}{ll}
M_{13}^{\prime} & M_{14}^{\prime} \\
M_{23}^{\prime} & M_{24}^{\prime}
\end{array}\right), \\
C=\left(\begin{array}{ll}
M_{31}^{\prime} & M_{32}^{\prime} \\
M_{41}^{\prime} & M_{42}^{\prime}
\end{array}\right), & D=\left(\begin{array}{ll}
M_{33}^{\prime} & M_{34}^{\prime} \\
M_{43}^{\prime} & M_{44}^{\prime}
\end{array}\right) .
\end{array}
$$

The spectral function for the total and relative phases are given by

$$
\begin{aligned}
& A_{T}(\mathbf{q}, \omega) \equiv \frac{1}{\pi} \operatorname{Im}\left\langle\theta_{1}^{*} \theta_{1}\right\rangle=\frac{1}{\pi} \operatorname{Im}\left(M^{\prime \prime-1}\right)_{11}\left(\mathbf{q}, \omega+i 0^{+}\right), \\
& A_{R}(\mathbf{q}, \omega) \equiv \frac{1}{\pi} \operatorname{Im}\left\langle\theta_{2}^{*} \theta_{2}\right\rangle=\frac{1}{\pi} \operatorname{Im}\left(M^{\prime \prime-1}\right)_{22}\left(\mathbf{q}, \omega+i 0^{+}\right),
\end{aligned}
$$

where $A_{T}(\mathbf{q}, \omega)$ and $A_{R}(\mathbf{q}, \omega)$ correspond to the total and relative phase fluctuations, respectively [38,39]. 
[1] I. Bloch, J. Dalibard, and W. Zwerger, Rev. Mod. Phys. 80, 885 (2008).

[2] M. Lewenstein et al., Adv. Phys. 56, 243 (2007).

[3] T. Oka and H. Aoki, Phys. Rev. B 79, 081406 (2009).

[4] W. Zheng and H. Zhai, Phys. Rev. A 89, 061603 (2014).

[5] G. Jotzu, M. Messer, R. Desbuquois, M. Lebrat, T. Uehlinger, D. Greif, and T. Esslinger, Nature (London) 515, 237 (2014).

[6] B. K. Stuhl, H. I. Lu, L. M. Aycock, D. Genkina, and I. B. Spielman, Science 349, 1514 (2015).

[7] R. B. Laughlin, Phys. Rev. Lett. 50, 1395 (1983).

[8] S. C. Zhang, T. H. Hansson, and S. Kivelson, Phys. Rev. Lett. 62, 82 (1989).

[9] J. K. Jain, Phys. Rev. Lett. 63, 199 (1989).

[10] X. G. Wen, Int. J. Mod. Phys. B 6, 1711 (1992).

[11] T. Neupert, L. Santos, C. Chamon, and C. Mudry, Phys. Rev. Lett. 106, 236804 (2011).

[12] E. Tang, J. W. Mei, and X. G. Wen, Phys. Rev. Lett. 106, 236802 (2011).

[13] K. Sun, Z. Gu, H. Katsura, and S. Das Sarma, Phys. Rev. Lett. 106, 236803 (2011).

[14] X. L. Qi and S. C. Zhang, Rev. Mod. Phys. 83, 1057 (2010).

[15] M. Z. Hasan and C. L. Kane, Rev. Mod. Phys. 82, 3045 (2010).

[16] Z. Wang, X. L. Qi, and S. C. Zhang, Phys. Rev. Lett. 105, 256803 (2010).

[17] V. Gurarie, Phys. Rev. B 83, 085426 (2011).

[18] D. Zheng, G. M. Zhang, and C. Wu, Phys. Rev. B 84, 205121 (2011).

[19] M. Hohenadler, Z. Y. Meng, T. C. Lang, S. Wessel, A. Muramatsu, and F. F. Assaad, Phys. Rev. B 85, 115132 (2012).

[20] C. Hickey, P. Rath, and A. Paramekanti, Phys. Rev. B 91, 134414 (2015).

[21] W. Zheng, H. Shen, Z. Wang, and H. Zhai, Phys. Rev. B 91, 161107 (2015).

[22] J. He, S. P. Kou, Y. Liang, and S. Feng, Phys. Rev. B 83, 205116 (2011).

[23] J. He, Y. H. Zong, S. P. Kou, Y. Liang, and S. Feng, Phys. Rev. B 84, 035127 (2011).
[24] J. Maciejko and A. Ruegg, Phys. Rev. B 88, 241101(R) (2013).

[25] Y. Liang, J. He, Y.-J. Wu, Y.-X. Zhu, and S.-P. Kou, Eur. Phys. J. B 86, 466 (2013).

[26] X. J. Liu, K. T. Law, and T. K. Ng, Phys. Rev. Lett. 112, 086401 (2014).

[27] BCS-BEC Crossover and the Unitary Fermi Gas, edited by W. Zwerger, Lecture Notes in Physics Vol. 836 (Springer, Berlin, 2012).

[28] F. D. M. Haldane, Phys. Rev. Lett. 61, 2015 (1988).

[29] D. J. Thouless, M. Kohmoto, M. P. Nightingale, and M. den Nijs, Phys. Rev. Lett. 49, 405 (1982).

[30] P. Fulde and R. A. Ferrell, Phys. Rev. 135, A550 (1964).

[31] A. I. Larkin and Y. N. Ovchinnikov, Zh. Eksp. Teor. Fiz. 47, 1136 (1964) [Sov. Phys. JETP 20, 762 (1965)].

[32] Y. Xu, R. L. Chu, and C. Zhang, Phys. Rev. Lett. 112, 136402 (2014).

[33] Y. Cao, S. H. Zou, X. J. Liu, S. Yi, G. L. Long, and H. Hu, Phys. Rev. Lett. 113, 115302 (2014).

[34] H. Hu, L. Dong, Y. Cao, H. Pu, and X. J. Liu, Phys. Rev. A 90, 033624 (2014).

[35] A. P. Schnyder, S. Ryu, A. Furusaki, and A. W. W. Ludwig, Phys. Rev. B 78, 195125 (2008).

[36] S. Ryu, A. Schnyder, A. Furusaki, and A. Ludwig, New J. Phys. 12, 065010 (2010).

[37] Y. J. Wu, N. Li, and S. P. Kou, Eur. Phys. J. B 88, 255 (2015).

[38] J. R. Engelbrecht, M. Randeria, and C. A. R. Sade Melo, Phys. Rev. B 55, 15153 (1997).

[39] E. Zhao and A. Paramekanti, Phys. Rev. Lett. 97, 230404 (2006).

[40] R. B. Diener, R. Sensarma, and M. Randeria, Phys. Rev. A 77, 023626 (2008).

[41] J. M. Vogels, K. Xu, C. Raman, J. R. Abo-Shaeer, and W. Ketterle, Phys. Rev. Lett. 88, 060402 (2002).

[42] Y. G. Ponomarev et al., Solid State Commun. 129, 85 (2004).

[43] G. Blumberg, A. Mialitsin, B. S. Dennis, M. V. Klein, N. D. Zhigadlo, and J. Karpinski, Phys. Rev. Lett. 99, 227002 (2007).

[44] D. Mou, R. Jiang, V. Taufour, R. Flint, S. L. Budko, P. C. Canfield, J. S. Wen, Z. J. Xu, G. Gu, and A. Kaminski, Phys. Rev. B 91, 140502(R) (2015). 